\title{
Tip-sample interactions in atomic force microscopy: I. Modulating adhesion between silicon nitride and glass
}

\author{
Jan H Hoh†§, Jean-Paul Revel† and Paul K Hansmał \\ tDivision of Biology, California Institute of Technology, Pasadena, CA 91125. \\ USA \\ ‡Department of Physics, University of California Santa Barbara, Santa Barbara, \\ CA 93117, USA
}

Received 9 November 1991, accepted for publication 16 March 1992

\begin{abstract}
An adhesive interaction between a silicon nitride AFM tip and glass substrate in water is described. This adhesion is in the range $5-40 \mathrm{nN}$, of which a large component is likely to be due to hydrogen bonding between the silanol groups on both surfaces. The interaction can be modulated by a variety of buffers commonly used in biochemical and biological research, including sodium phosphate, tris (hydroxymethyl)aminomethane, glycine, and N-2-hydroxyethylpiperazine $\mathrm{N}^{\prime}$-2-ethanesulfonic acid. Using these buffers it appears that there are effects of ion concentration, ion type, and $\mathrm{pH}$ on the measured adhesion. Of the conditions examined, phosphate was most effective at reducing adhesion and could be used at concentrations as low as $10 \mathrm{mM}$ at neutral $\mathrm{pH}$. The results demonstrate that the chemical interactions between tip and sample can be modulated, and provide a basis for designing conditions for imaging and manipulating biologica! molecules and structures.
\end{abstract}

\section{Introduction}

The atomic force microscope (AFM) is a new tool for high resolution imaging of surfaces (Binnig et al 1986). We have for sometime been interested in applying this new technology to the imaging of biological macromolecules, cellular organelles and whole cells. Initial efforts have been very encouraging (Drake et al 1989, Gould et al 1990, Butt et al 1990, 1991, Hoh et al 1991), however, the nature of the interaction between tip and sample remains poorly understood. We have begun a series of experiments to evaluate tip-sample interactions and how they can be modulated. In the present paper we describe the modulation of interactions between a silicon nitride tip and a glass surface, often used as a substrate for biological material, in an aqueous environment.

In normal imaging mode, the AFM scans the tip or sample in the $x$ and $y$ directions while monitôring topography. The commercial AFM we have used is also equipped with a force mode of operation, in which the $x$ and $y$-scan directions are disabled and the sample is scanned only in $z$. When operating in this mode, the sample starts out away from the cantilever tip and is

§Present address: Department of Physics, University of California, Santa Barbara, CA 93117 , USA advanced toward the tip until it makes contact. After moving some distance in contact, the stage is retracted until the tip leaves the surface. Data from the scan is displayed as a graph of cantilever deflection versus sample position. Since cantilever deflection is proportional to force applied, this is often referred to as a force versus distance curve and will here be called simply the force curve. It should be noted that these curves are very different from force versus distance curves obtained from other instruments, such as the surface force apparatus, that display interaction forces versus separation distance. Force curves are extremely sensitive to interactions between the tip and sample and have been used to measure forces between several different surfaces (Weisenhorn et al 1989, Burnham and Colton 1989, Weisenhorn et al 1991, Ducker et al 1991). Here we have used the force mode of operation to examine adhesive tip sample interactions in aqueous solutions.

\section{Experimental details}

A NanoScope II scanning probe microscope (Digital Instruments, Santa Barbara, CA, USA) with an AFM stage and glass fluid cell were used. Cantilevers were standard 
silicon nitride $\mathrm{V}$ shaped (Digital Instruments), with spring constants of $0.2 \mathrm{~N} \mathrm{~m}^{-1}$ (Albrecht et al 1990). Circular $(12 \mathrm{~mm}$ diameter) glass cover slips were obtained from Fisher Scientific (Pittsburgh, PA, USA), and chemicals were obtained from Fisher Scientific or Sigma Chemical Company (St. Louis, MI, USA).

Solutions were all prepared from Milli-Q (Waters Millipore, Bedford, MA, USA) purified water $(>10 \mathrm{M} \Omega$ ). The $\mathrm{pH}$ of different solutions were adjusted with aqueous $\mathrm{HCl}$ or $\mathrm{NaOH}$. Phosphate buffered saline (PBS) was $13 \overline{\mathrm{mM} \mathrm{NaCl}}, \quad 2.7 \mathrm{mM} \mathrm{KCl}, \quad 1.5 \mathrm{mM} \mathrm{K}^{2}{ }_{2} \overline{P O}_{4}$, and $4.3 \mathrm{mM} \mathrm{Na}_{2} \mathrm{HPO}_{4}$ at $\mathrm{pH}$ 7.2. Glass cover slips were mounted on stainless steel stubs with epoxy, etched briefly with dilute $\mathrm{HF}$ to expose a clean surface, and rinsed for several minutes in water. Cover slips prepared this way were usually used the same day they were prepared.

In a typical experimental series, a tip was brought into contact with a glass cover slip in water, and the scanning of the stage was initiated. We routinely used a scan range of $200-400 \mathrm{~nm}$, at $25 \mathrm{~Hz}$. The system was allowed to stabilize for 5-10 minutes and the adhesive force was determined by measuring the distance the cantilever moved past zero deflection when the sample was withdrawn from contact, as described previously (Weisenhorn et al 1989, 1991). The solution in the cell was then changed by flushing with $\simeq 10 \mathrm{ml}$ of the test solution at approximately $2 \mathrm{ml} \mathrm{s}^{-1}$. This was allowed to equilibrate for several minutes and the adhesive force was determined as before. The cell was then flushed with water again and the original interaction was restored before a second measurement was taken.

\section{Results and discussion}

In pure water the tip exhibited a strong adhesion with the glass of $5-40 \mathrm{nN}$, with a great deal of variability between experiments (figure 1(a)). The source of this variability is not fully understood, though changes in contact area because of differencens in tip geomemetries and variationons in the surface provide the most likely explanation. Consistent with this suggestion, it was observed that the adhesive force in water would often increase gradually during long experiments (3-10 h), suggesting that the tip was becoming duller and the contact area with the surface increased. This presents the possibility of using adhesive interactions with well-characterized surfaces for determining tip sharpness, a problem that has not been amenable to standard microscopies. It is also possible that the gradual increase resulted from solvation of either of the surfaces.

The tip-glass adhesion was highly sensitive to $\mathrm{pH}$ and reduced below the limits of detection (roughly $10^{-10} \mathrm{~N}$ in these experiments) at $\mathrm{pH}$ greater than $\simeq 9$ (Figure 1(b), figure 2). At high $\mathrm{pH}$ the force curve was highly repulsive approaching and withdrawing, indicating that both surfaces had become strongly charged and the presence of a substantial double-layer force.

A mechanism for this adhesion must be a function of the two surfaces interacting and take into account the

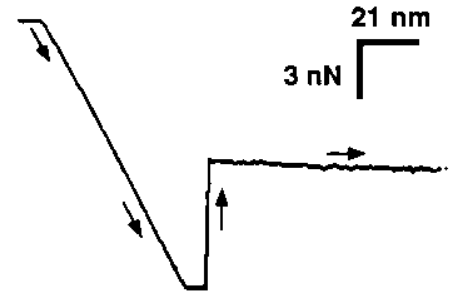

(a)

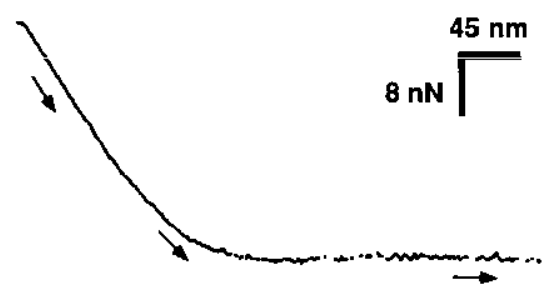

(b)

Figure 1. Force curves for a silicon nitride tip on glass. (a) At low pH (5) a large adhesive force was observed. Only the trace for the stage retracting is shown and the bottom of the curve is off scale. $(b)$ At high $\mathrm{pH}(>9)$ the interaction between the surfaces was highly repulsive. No adhesion is seen.

fact that the forces observed are larger than would be expected for van der Waals interactions (Weisenhorn et al 1989, 1991). The chemistry of silicon nitride is complex, though it is known that chemical vapor deposited silicon nitride, the material the AFM tips are made of, has roughly 5 hydroxyl groups (silanols) per $\mathrm{nm}^{2}$ (Harme et al 1987 , Bousse and Mostarshed 1991), similar to most glasses. Further the hydroxyl groups on the two surfaces have $\mathrm{p} K_{\mathrm{a}} \mathrm{s}$ for the dissociation of $\mathrm{SiOH}$ to $\mathrm{SiO}^{-}$of about 6-7 (Harme et al 1987, Doremus 1973). However, it should be noted that as the two surfaces are brought together the $\mathrm{p} K_{\mathrm{a}} \mathrm{s}$ of the hydroxyl groups will rise making it impossible to determine the exact protonation states. The chemical nature of the two surfaces, i.e. the high density of hydroxyl groups, the magnitude of the adhesive force and

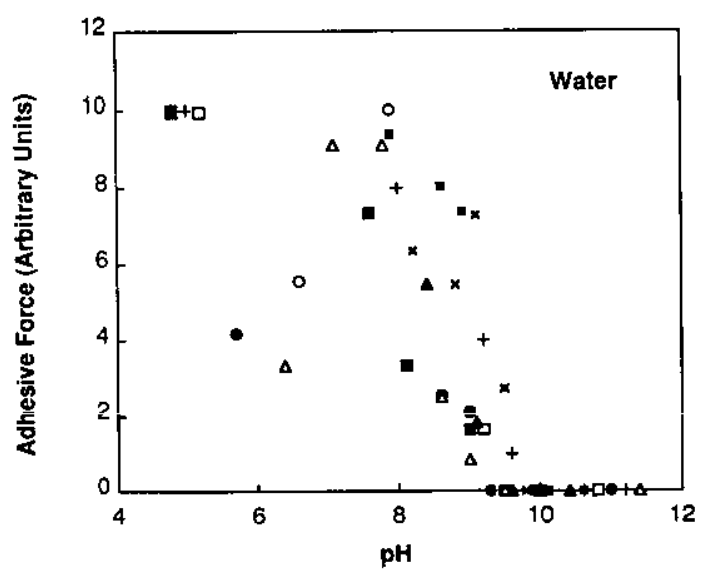

Figure 2. Titration of adhesive force between a silicon nitride tip and a glass surface in water. Because of the variability between experiments, usually $5-40 \mathrm{nN}$ at low $\mathrm{pH}$, the force values were all rescaled so the force at the lowest $\mathrm{pH}$ in a given experiment was 10 . Different symbols represent separate experiments. 


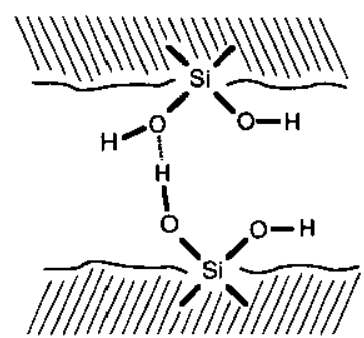

(a)

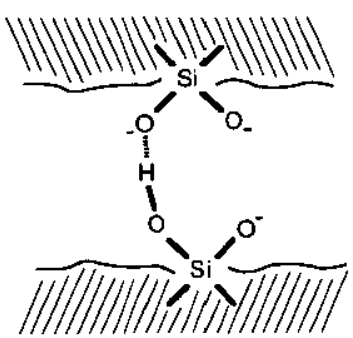

(b)
Figure 3. Schematic of the two predominant types of hydrogen bonds likely to form between an oxidized silicon nitride surface, and glass. (a) At low $\mathrm{pH}$ most hydroxyl groups are protonated resulting in little electrostatic repulsion, and a large number of bonds. $(b)$ At $\mathrm{pHs}$ over 9 , most of the hydroxyl groups are not protonated, even allowing for a shift upward of the hydroxyl group $\mathrm{p} K_{\mathrm{a}} \mathrm{s}$ as the surfaces are brought together, resulting in electrostatic repulsion between the surfaces and very few bonds. However, these bonds are of the 'strong' type, as opposed to the weak type in (a) (Jefferey and Saenger 1991).

the sensitivity of the adhesion to $\mathrm{pH}$ suggest that a significant fraction of the adhesion observed between the silicon nitride tip and glass may be due to hydrogen bonding (figure 3). Consistent with the hydrogen bonding hypothesis, we have recently resolved discrete steps in the adhesive interaction described here (unpublished observation).

A common biological buffer system, PBS, also reduced adhesion below detection limits, but at near neutral pH (7.2). Each component of this buffer was tested individually for its effect on adhesion. Sodium or potassium ions in combination with chloride ions did slightly reduce the measured adhesion, but concentrations up to $1 \mathrm{M}$ did not eliminate adhesion at low $\mathrm{pH}$. Titration of $10 \mathrm{mM}$ sodium phosphate revealed that it alone eliminated adhesion near neutral pH (figure $4(a)$ ), suggesting that phosphate was primarily responsible for reduction of adhesion with pBs. This effect was also concentration dependent, and concentrations lower than $10 \mathrm{mM}$ were not effective at $\mathrm{pH} 7.2$ (figure $4(b)$ ). The effect of $100 \mathrm{mM}$ sulfate, another multivalent anion, was also examined up to $\mathrm{pH} \mathrm{6,} \mathrm{where} \mathrm{it} \mathrm{is} \mathrm{essentially} \mathrm{completely} \mathrm{dissociated,} \mathrm{but}$ no significant change in adhesion was observed. These results suggest that in addition to $\mathrm{pH}$, there is also a specific effect for phosphate ions and a concentration effect. The mechanism by which sodium phosphate acts is not understood, however in principle it could modify properties of the medium or bind directly to either of the surfaces.

Several other buffers including tris(hydroxymethyl) aminomethane (Tris), glycine, and N-2-hydroxyethylpiperazine $\mathrm{N}^{\prime}$-2-ethanesulfonic acid (Hepes) were also examined. None of these was as effective as phosphate in reducing adhesion at low concentration. However at $100 \mathrm{mM}$, they all had an effect. Hepes eliminated adhesion at neutral $\mathrm{pH}$, while glycine was effective near $\mathrm{pH} 8$, as was Tris (figure 5).

When the AFM is used for imaging in contact mode, it is important to reduce interactions that distort or (a)

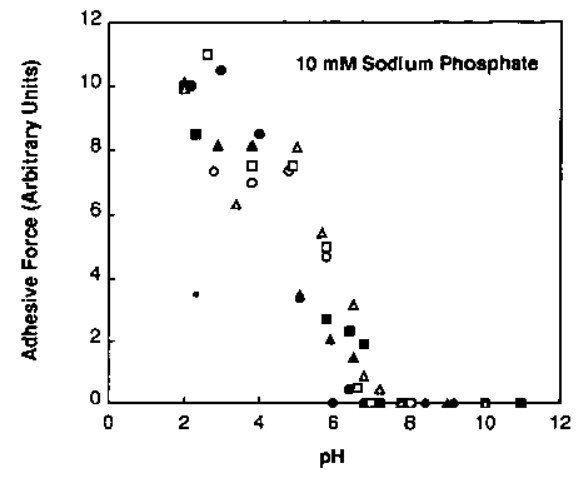

(b)

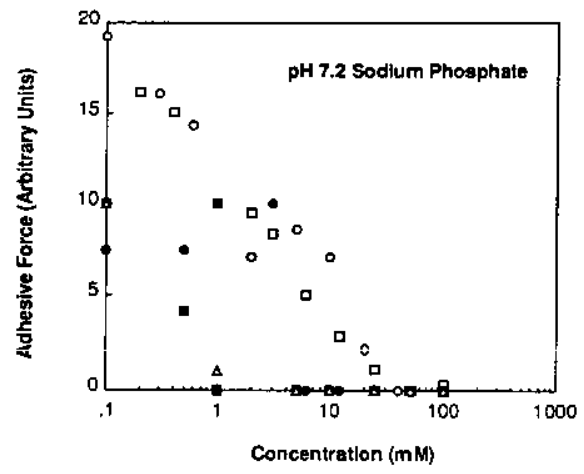

Figure 4. (a) Titration of adhesion between a silicon nitride tip and glass as a function of the $\mathrm{pH}$ of $10 \mathrm{mM}$ sodium phosphate. (b) Effect of sodium phosphate concentration at $\mathrm{pH} 7.2$ on adhesion. Values were rescaled as in figure 2 and different symbols represent separate experiments.

damage the sample, particularly in the case of biological materials that are often soft. The main approach to this has been to minimize the force applied to the sample by using cantilevers with smaller spring constants or by imaging in liquids. However, as described here, actual bond formation or other strong interactions between the tip and sample may occur in some circumstances. This will have the effect of pulling on the sample, until the bonds break or the sample is dislodged from the substrate, as the tip moves across the surface. PBS appears to substantially reduce adhesion between silicon nitride and glass, and also between silicon nitride and at least one type of biological membrane (isolated gap junctions, unpublished observation). PBS also contains a high concentration of $\mathrm{NaCl}$ which will have the effect of screening out most electrostatic interactions. Therefore PBS is a good medium for imaging biological material. If a low salt environment is needed, $10-20 \mathrm{mM}$ sodium phosphate at $\mathrm{pH} 7.2$ would work well, and if phosphate is undesirable, as is often the case, either Tris, Hepes or glycine are effective at different $\mathrm{p} \overline{\mathrm{H}} \mathrm{s}$, at $100 \mathrm{mM}$.

The AFM has also been used to manipulate structures such as Langmuir-Blodgett films (Hansma et al 1991), gap junctions (Hoh et al 1991), and DNA (Hansma 1991 private communication). For this application it may be important to vary interactions with the sample. The results described here suggest that this may be accomplished dynamically by changing the fluids in the cell. It may for example be possible to increase interactions, use 
(a)

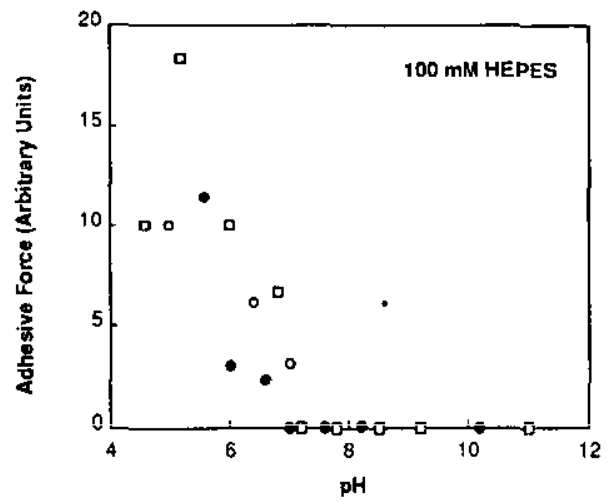

(b)

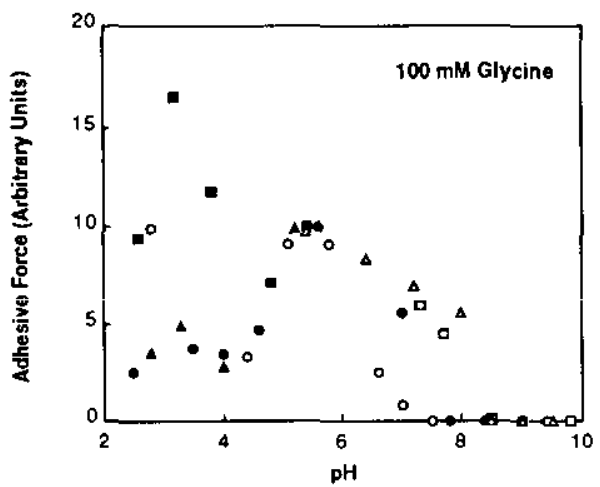

(c)

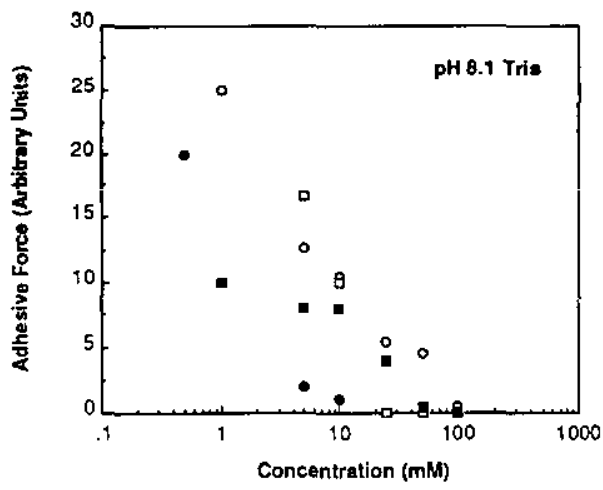

Figure 5. Titration of adhesion between a silicon nitride tip and glass as a function of the $\mathrm{pH}$ of (a) $100 \mathrm{mM}$ glycine and (b) $100 \mathrm{mM}$ Hepes. (c) Effect of concentration of Tris at $\mathrm{pH} 8.1$ on adhesion. Values were rescaled as in figure 2 and different symbols represent separate experiments.

the tip to 'dissect' a gap junction membrane, and reduce interactions for imaging, rather than varying applied force as previously described (Hoh et al 1991). Another, more general possibility, would be to pick up a structure with the tip and deposit it on a surface by modulating interactions.

\section{Conclusion}

Here we have described experimental evidence for an adhesion based on the formation of hydrogen bonds between silicon nitride and glass, that can be modulated by $\mathrm{pH}$, specific ions, and concentration. These observations have practical implications for designing conditions to image and manipulate structures with the AFM.

\section{Acknowledgments}

This research was supported by the Office of Naval Research (PKH) and fellowships from the Colvin and Merck funds $(\mathrm{JHH})$.

\section{References}

Albrecht T R, Akamine S, Carver T E and Quate C F 1990 J. Vac. Sci. Technol. A 8 3386-96

Binnig G, Quate C F and Gerber C 1986 Phys. Rev. Lett. 56 930-3

Bousse L and Mostarshed S 1991 J. Electroanal. Chem. 302 269-74

Butt H-J, Downing K H and Hansma P K 1990 Biophys. J. $58 \quad 1473-80$

Butt H-J, Wolf E K, Gould S A C and Hansma P K $1991 \mathrm{~J}$. Struct. Biol. 105 54-61

Burnham N A and Colton R J 1989 J. Vac. Sci. Technol. A7 2906

Drake B, Prater C B, Weisenhorn A L, Gould S A C, Albrecht T R, Quate C F, Cannell D S, Hansma H G and Hansma P K 1989 Science 243 1586-9

Doremus R H 1973 Glass Science (New York: Wiley) pp 349

Ducker W A, Senden T J and Pashley R M 1991 Nature 353 $239-41$

Gould S A C et al 1990 J. Vac. Sci. Technol. A8 369-73

Harme D L, Bousse L J, Shott J D and Meindl J D 1987 IEEE Trans. Electron Devices ED-34 1700-7

Hansma H G, Gould S A C, Hansma P K, Gaub H E, Longo M L and Zasadzinski J A N 1991 Langmuir 7 1051-4

Hoh J H, Lal R, John S A, Revel J P and Arnsdorf M F 1991 Science 253 1405-8

Jeffrey G A and Saenger W 1991 Hydrogen Bonding in Biological Structures (Berlin: Springer) pp 569

Weisenhorn A L, Hansma P K, Albrecht T R and Quate C F 1989 Appl. Phys. Lett. 54 2651-3

Weisenhorn A L, Maivald P, Butt H-J and Hansma P K 1992 Phys. Rev. B at press 